\title{
ENERGY EFFICIENT CENTRALIZED DYNAMIC CLUSTERING RESERVATION MAC PROTOCOL FOR MWSN
}

\author{
Suma. $\mathbf{G}^{\mathbf{1}}$ and Siddappa. $\mathbf{M}^{\mathbf{2}}$ \\ ${ }^{I}$ Department of Telecommunication Engineering, Sri Siddhartha Academy of Higher Education, India \\ ${ }^{2}$ Department of Computer Science and Engineering, Sri Siddhartha Institute of Technology, India
}

\begin{abstract}
Mobile wireless sensor networks (MWSN) have recently emerged as a hot research topic as their communication links and topology change due to node mobility. In a dynamically changing environment it is essential to build energy efficient communication routes with least communication overheads. Energy is one of the most important concerns for MWSNs. To have a long lasting network lifetime, we propose a centralized, periodic, selective sensing and dynamic clustering algorithm for delay tolerant and high integrity MWSNs, aimed to enhance the connectivity and performance. All dynamically varying parameters such as location, number of nodes present in each virtual grid, residual energy are considered while forming clusters and selecting cluster head $(\mathrm{CH})$. Reclustering and selection of $\mathrm{CH}$ is centralized and performed by sink. Cluster size is not constant and may vary from cluster to cluster in every round. If nodes in any grid is less, then nodes of neighboring grids are clubbed to form clusters. Reclustering is done in every round and to avoid data duplication, some nodes in every cluster are selected based on proximity and are not allowed to sense and transmit in that round. It is assumed in a close vicinity if more than one sensors are present they all generate same data as they sense the same physical quantity, this reduce redundant data generation sensing is performed only by selected nodes. Inter and intra communication slots are reserved depending on size of cluster by sink in every round. The algorithm is evaluated using stationary and mobile sink.
\end{abstract}

\section{Keywords:}

MWSN, Dynamic Clustering, Mobile Sink, Dynamic TDMA Reservation, Selective Sensing

\section{INTRODUCTION}

The distribution of Sensors are remotely done in large numbers and conventionally operate independently in unmanned environments. As MWSN comprises of large number of mobile sensor nodes with limited storage space, radio communication capabilities, bandwidth, and energy are deployed in a geographical area. Sensor energy depletes while performing activities like sensing, data processing, communication and mobility. Extending the network lifetime is the main concern in any MWSN.

Clustering in MWSN can reduce energy dissipation and improve scalability. Nodes are usually grouped into independent clusters. Each cluster has a head, often designated as cluster head $(\mathrm{CH}) . \mathrm{A} \mathrm{CH}$ is authorized to create synchronization among cluster nodes, collect data from its members, and to create a back bone network to forward sensed data to sink. A member node consumes less power from battery than that by $\mathrm{CH}$. One of the important parameter considered for network evaluation is its lifetime. Clustering and $\mathrm{CH}$ selection affects network lifetime leading them to be energy efficient [1].
In mobile wireless sensor networks to enhance the quality of service hierarchical clustering schemes have been widely used. Node mobility dynamically influences the topology of the clustered network in MWSN, this in turn affects the separation and compactness of the clusters formed. Reclustering and route adjustments are to be done in every round to overcome the influence of mobility in nodes. Reclustering is not done very often in static sensor network as topology changes are very minimum and occur when nodes die due to its battery being fully used up or sensor gets destroyed causing link variation. This is not the case in MWSN which requires reclustering time and again due to frequent topology changes caused by node mobility causing more energy loss. Nodes spend their energy for sensing, data processing and communication (transmitting and receiving). Node energy spent on sensing is least, but if sensing is done selectively can reduce generation of redundant data, in turn reducing aggregation process and intra communication load saving nodes energy and network's resource. Energy spent by nodes for communication is directly related to size of data and distance for which it is transferred. Leading to select $\mathrm{CH}$ almost at the center of cluster. Data generation is not very frequent in many MWSN applications such as weather report of remote areas. Many applications may also not require high throughput and can tolerate latency but requires high integrity. Such applications need high energy efficiency as battery replacement is not easily possible. To enhance nodes battery life they are allowed to perform minimum and essential works only by selectively allowing them to sense. In this work clustering is centrally performed, inter and intra communication slots are reserved by sink, this makes the algorithm collision free, reduces retransmissions and improves nodes life and in turn increase network longevity.

This work comprises of two clustering can be of two types, based on where clustering decision is taken

- CH-clustering (distributed) and

- Sink clustering (centralized).

In former member nodes and $\mathrm{CH}$ selection is done locally. In static networks cluster size is fixed hence all clusters have same time allotment for intra and inter communication. In MWSN if fixed cluster size is used many nodes may be left out without joining any cluster as they may not find sufficient nodes to form a cluster. This increases the number of single member clusters which sense and transmit independently to sink. Therefore cluster size cannot be fixed when topology changes frequently. Selecting nodes for sensing process and CHs selection is decided by sink hence called as centralized clustering. It is observed centralized clustering with selective sensing and MAC reservation improve the performance of proposed protocol better. The proposed protocol employs single wireless channel, creates clusters, identifies $T x$ nodes within each cluster and coordinated by sink. 
Thus resulting in improving networks performance and prolonging life time of MWSNs.

This work is carried out in four stages. In first stage clusters are re clustered in every round and their size is not fixed. If any grid has less sensors than the threshold value, then sensors from adjoining grids are clubbed to create a new cluster. This reduces number of CHs formed in every round, as nodes which are left out are to be allowed to perform as single node cluster. The second stage selects $\mathrm{CH}$ for each cluster to facilitate intra and inter-cluster communication. This selection is based on residual energy, node position and mobility. The third stage addresses data redundancy by selecting only one node when more than one node is present in close vicinity. In fourth stage, sink creates reservation schedule for clusters intra and inter communication based on present cluster size in that round. The impact of all these on network life time is compared using stationary and mobile sink.

The rest of this article is outlined as follows: Section 2 provides reports some related works in brief, section 3 introduces the proposed dynamic clustering and reservation scheme, section 4 provides the results and discussion and section 5 concludes the work.

\section{RELATED WORKS}

Wireless sensor network's performance is assessed by measuring some important parameters such as throughput, energy consumption, latency and overhead [12].

Throughput: Quantity of sensed data bytes/time transmitted from sensor nodes to base station.

Energy Consumption: Amount of Energy used from the battery that powers the sensor node.

Latency: Time taken by a packet to travel from sensor node to base station, sometimes called as delay.

Overhead: Total number of control packets other than pay load transmitted from source to sink [15].

Network Lifetime: The time required for the last node to lose its energy fully or till the living nodes are unable to connect to sink.

It is believed that clustering effectively improves the performance of wireless sensor network, such as energy consumption and data transmission efficiency. In the past few years, a large number of clustering schemes are proposed.

Leach [2] is an energy-efficient adaptive clustering protocol, cluster headers are randomly selected among the nodes other nodes join nearest cluster, where $\mathrm{CHs}$ is periodically rotated. In [3], Younis et al. propose HEED, clustering CHs, it follows an iterative procedure to create clusters and nominate their head. $\mathrm{CHs}$ are rotated in accordance with their residual energy. Considering node mobility in MWSNs. In [4], Getsy et al. used a routing algorithm along a clustering method. $\mathrm{CH}$ selection is performed considering amount of remaining energy, less speed and good coverage range. Energy based selection mechanism and the maximum remaining energy in node estimation technique implemented betters routing energy performance.

Anitha et al. [5] used EEDBC-M protocol to enhance LEACH-mobile with a better cluster formation process. In a clustered MWSN, random node mobility dynamically influences the topology of the network. In a changing topology it may be not optimal for a node to get attached to current cluster though its communication distance is increasing, which results in extra energy consumption on transmission and reduces packet delivery ratio. Clustering in more than one level and presents a new residual energy difference ratio for selecting cluster head. To overcome mobility problems, considers both node and systems remaining energy to calculate energy ratio in every clustering period. This ratio is used in selecting $\mathrm{CH}$. Hence algorithm is energy efficient [6]. In [7] Ma et al. performs dynamic clustering considering remaining energy rate.

This is calculated using nodes and systems average remaining energy. This avoids nodes with less left over energy getting selected as cluster head. Hence improves network lifetime, overall throughput and battery efficiency of mobile sensor networks. In [8], et al. proposes Cluster head selection scheme. The criteria for cluster head selection are energy level and the similarity of movement. To cope with mobility, proposes a dynamic slot allocation for reducing the unused slots based on the predicted residence time between the cluster head and the members. The proposed clustering scheme prolongs the network lifetime.

In [9], Zuniga, et al. states reclustering process helps in assigning nodes to optimal clusters and improves the service quality. Re-clustering schemes can be mainly centralized or distributed in nature. Sink or $\mathrm{CHs}$ performs reclustering in case of centralized schemes. In [10] scheduling mechanism for collaborating sensors and attain error-bound scheduling control to monitor applications is utilized and the advantages of both methods to strike a compromise between energy consumed and prediction accuracy. Sensing planned based on two error information, a) error calculated locally b) error predicted by neighboring nodes. Sensors are switched on when user mentioned error limit is crossed.

In [11], Gu et al. separates switching and scheduling process from one another. Switching preformed at node level while scheduling is performed separately on computational entity and this reduces area breaching. Present a hybrid architecture for duty cycled sensor networks. Which blends three ideas: Asymmetric Architecture, Generic Switching and Global Scheduling. Working parameters for every node is calculated by scheduling and nodes are turned on and off based scheduling parameters.

In [12] Guimaraes et al. presents collision free MAC protocol using on off controlled TDMA for sensor network. Energy consumption is low and high data throughput. Uses carrier sensing to access the single channel present channel. In [13], Xie et al. states, Compressive sensing (CS) can reduce the number of data transmissions and balance the traffic load throughout networks. Centralized clustering uses hybrid CS for sensor networks. The sensor nodes are arranged into clusters and raw data is transmitted to cluster head. $\mathrm{CH}$ uses compressive method to compress data and back bone network is used to send projections to sink. In, [16] mobile sink is a good scheme to balance nodes energy utilization and puts forward rules that maintains optimal route to sinks latest location with minimum route adjustments. 


\section{OVERVIEW OF CENTRALIZED DYNAMIC CLUSTERING RESERVATION MAC SCHEME}

In this paper, we propose a new clustering protocol for mobile wireless sensor networks which is collision free, dynamic, and with centralized reservation MAC protocol, having high throughput and energy efficiency. All the nodes are assumed to be homogenous in nature. Each round can be broadly classified into two phases, cluster formation and communication phase. Initially sink collects information about nodes position and residual energy from nodes. Creates clusters and selects cluster head for every cluster. Selects few nodes in every cluster and allows only them to sense and transmit by declaring them as $T x$ nodes (Transmitting node) rest nodes are not allowed to sense and are set to No_Tx, for that round. Sink Selects $T x$ nodes by selective sensing algorithm within each cluster and sets intra communication schedule for only them. Tx (Transmitting nodes) sense the data and transmits to $\mathrm{CH}$ one by one following preset communication schedule. This is repeated for all clusters. After all $T x$ nodes data is received, $\mathrm{CHs}$ transmits it to sink. At the end of every round re clustering performed to cope with continuous changes in topology. Above steps are repeated till there are enough number of nodes to carry out hop by hop communication from $\mathrm{CHs}$ to sink.

\subsection{OBJECTIVE}

Focus is on reducing redundant data generation by selective sensing, reduce number of left out nodes which are unable to join cluster by setting lower and upper threshold for cluster size, minimize collision and reduce the retransmissions by reserving time slots for intra and inter cluster communication, Mobile sink is also used to extend network life with minimum overheads.

The paper describes implementation of

1. Selective sensing,

2. Centralized dynamic reclustering,

3. Reserved dynamic time slots and

4. Mobile sink (MS).

\subsection{CENTRALIZED DYNAMIC CLUSTERING}

$\mathrm{CH}$ is selected by sink and not elected by nodes, clusters are formed within grids hence evenly distributed,resources are well managed. Single node clusters are reduced. Centralized clustering conserves CHs' energy that would be used for forming clusters and electing $\mathrm{CHs}$. As cluster members assignment and selection of $\mathrm{CH}$ is performed by sink which has unlimited energy.

\subsection{SELECTIVE SENSING}

Reduce the amount of redundant data generated by sensors when many nodes are present in close vicinity. If all of them are allowed to sense they generate redundant data. Leading to need for data aggregation by $\mathrm{CH}$. This algorithm selects nodes in every cluster in such a way that a minimum distance is maintained between sensing nodes, other nodes are not allowed to sense. The threshold distance between sensing nodes can be adjusted depending on the quality of data required. Hence avoids redundant data generation, lessens the amount of data generated and makes scheme lightweight and improves network life

\subsection{DYNAMIC RESERVATION}

Time allotment for sensing and communication is directly related to cluster size in that round and varies dynamically from round to round. Inter and intra communication is carried out in reserved slots set by sink, hence this protocol is centralized and Collision free.

\subsection{MOBILE SINK}

A mobile sink is used to collect data, this reduces $\mathrm{CH}$ to sink communication distance. Sink follows a fixed path inside the field following certain rules discussed in section 4 . It collects data and in its forward and as well as in return journey. MS reduces energy used up for inter cluster communication and betters algorithms' performance.

It is assumed that Sensing area is flat. Sensor nodes are homogenous in nature, all nodes have initial energy of $100 \mathrm{j}$ and sink has unlimited energy. Initial distribution of Sensor nodes is uniform in sensing area. Each sensor node is capable of identifying its location in sensing field with the help of GPS in them. Nodes are capable of measuring their own residual energy after every round. Receiver and transmitter dissipate 0.000001 mw and $0.000005 \mathrm{mw}$ respectively. It is assumed that radio can be turned on and off as required. Nodes follow Random bounded mobility and Pause Time model [14]. The performance of this algorithm is tested using stationary and mobile sink.

Implementation of work is mainly carried out two stages.

1. Cluster formation, has the following steps
a. Selection of $\mathrm{CH}$,
b. Clusters created from grids,
c. Selective sensing phase.
d. Dynamic time allotment.

2. Communication phase.

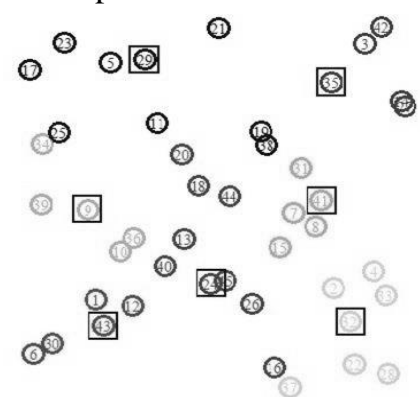

Fig.1. Random topology in $n^{\text {th }}$ round

\subsubsection{Cluster Formation:}

In this work, size of all grids is equal and of square shape, and formed by partitioning the sensing area. In Fig.1, Random topology of nodes in $N^{\text {th }}$ round is considered, here seven clusters are formed with varying cluster size, i.e. nodes in each cluster (including $\mathrm{CH}$ ) are in the range of 5 to 9 , shown in different colors. Every cluster consists of a $\mathrm{CH}$ represented by circle within square shaped box. The circle inside a square outside sensing area represents sink. 
At the beginning of every round sensor nodes update their residual energy and current position to sink. Sink checks if the number of sensor nodes inside each grid is greater than minimum threshold and less than maximum threshold value in every round then cluster is formed within one grid itself. Else grouping of adjacent grids done to create clusters, Fig.2. Sink decides the number of clusters and number of members to be assigned to each cluster in every round. Fig.4, for 25 rounds 19 to $24 \mathrm{CHs}$ are selected for a deployment of 200 nodes and 9 to $6 \mathrm{CHs}$ are selected for a deployment of 45 nodes in different random topology.

In Fig.2(a), total of 5 nodes are present in grid 1 they are node $9,25,30,38,41$. But grid 4 has only two nodes i.e. 22 and 39. A Cluster cannot be formed by considering only grid 4 , as it is less than minimum threshold required to form a cluster. Hence nodes of adjacent grids are clubbed to declare a cluster. Grid 4 nodes are combined with grid-1 nodes and one combined cluster is formed with seven nodes in yellow colour, as shown in Fig.2(b). This process continues until all the grids are scanned and all live nodes are included in one or the other cluster.

Sink selects one node as $\mathrm{CH}$ among the member nodes considering node parameters-Available energy, Mobility, Density. CHs sends Join_Request to members, willing members sends ACK and completes clustering.

\subsubsection{Selective Sensing Phase:}

If all cluster members are active and allowed to sense,then nodes in close vicinity generate same data,hence duplicate data will be forwarded to $\mathrm{CH}$. Selective sensing reduces $\mathrm{CHs}$ aggregation process and avoids duplicate data generation. From Fig. 5 it is found that for 200 deployed nodes only 72 to 100 are selected and set to $T x$ state in 25 rounds. For a deployment of 80 nodes, 32 to 44 nodes are set to $T x$ state in a deployment of 25 nodes. If more than one node is present in close vicinity, sink selects only one node with highest residual energy among them and sets it to Tx state (sensing and transmission). Other nodes within threshold distance are set to No_Tx state (no transmission). The distancesbetween nodes is calculated by Eq.(6). All these processes are controlled and monitered by sink in every round.

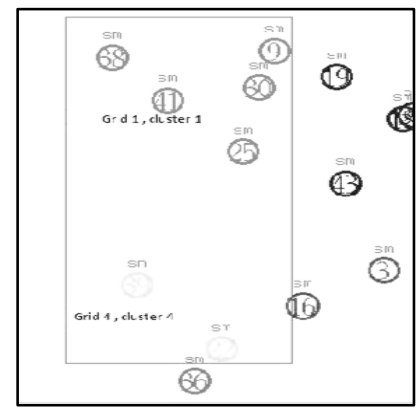

(a) In same cluster

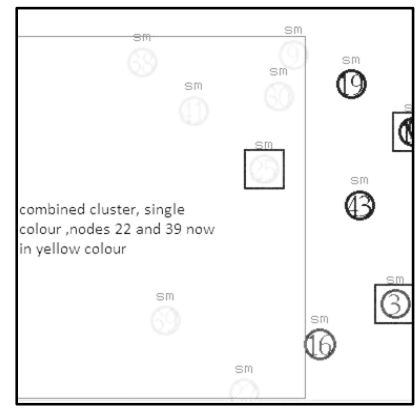

(b) From different cluster
Fig.2. Grouping of adjacent grids

In Fig.3, node 7 belonging to blue cluster and node 15 to red cluster are present in close visinity.Selective sensing stops node 15 from sensing as its position with respect to node 7 is less than threshold distance. Sets it No_tx state, as its residual energy is less than node 7 , This avoids the need of data aggregation of the received data at $\mathrm{CH}$. Hence selective saves energy by reducing the number of sensing nodes there by reducing the amount of data transmitted to CH. As per Eq.(9), $E_{T S-c l u s t e r}$ /round energy depletion depends on number of transmitting nodes in a cluster.

\subsubsection{Dynamic Time Allotment Phase:}

Cluster communication time allotment for intra and inter communication is dynamically decided by sink and informed to $\mathrm{CH}$ in every round. Intra cluster communication time is allocated on the number of nodes that are active in that round. $\mathrm{CH}$ instructs each node to go into No_t $x$ or be in $T x$ as per the schedule sent by sink. Nodes which are in $T x$ state sense and transmit data to $\mathrm{CH}$. This time mainly depends on number of members awake in that round. In Fig.2(b), Yellow cluster is greater than blue cluster, yellow has 9 nodes and all are in $T x$ state. Blue has 5 member and 4 are active and set to $T x$ state. Hence prior is allotted more time, Eq.(2).

As clustering is centralized and communication time scheduling decided by sink. Nodes are allowed to sense and transmit to $\mathrm{CH}$ in reserved timeslot as set by scheduler i.e. sink, therefore do not transmit redundant data as per Eq.(9). Total transmission energy used / cluster/ round, directly depends on number of $T x$ nodes in every cluster, if less nodes are in $T x$ state energy used is also less.

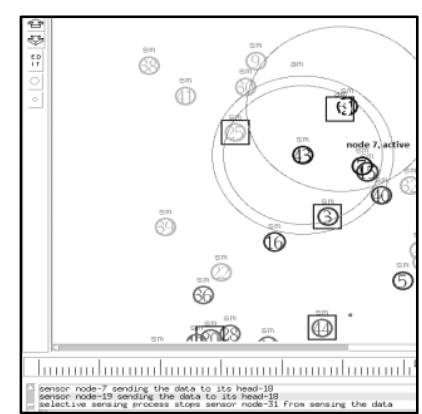

(a) In same cluster

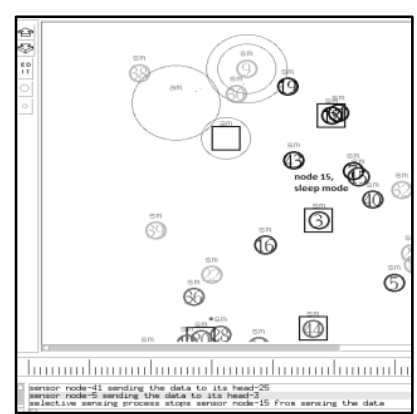

(b) From different cluster
Fig.3. Selective sensing

\subsubsection{Communication Phase:}

Reserved TDMA scheduling used for intra and inter data communication, this is single hop if sink is in radio range of $\mathrm{CH}$ else multi hop via cooperating $\mathrm{CHs}$ with shortest path towards sink. As cluster size and number of nodes allowed to sense and transmit keep changing from round to round as topology changes. The Fig. 5 shown number of reservations made for nodes in No_tx state.

Hence reservation schedule for communication depends on number of clusters and nodes in $T x$ state within each cluster. Sink carries out all these operations and reduces $\mathrm{CHs}$ load hence achieves high energy efficiency. The algorithm is tested placing sink outside and inside sensing field with stationary and mobile sink.

\subsubsection{Time Allotment: Network Lifetime}

Network lifetime, $T_{N E T}$ is calculated as,

$$
T_{N E T}=N_{R} \times t_{r}
$$

where, $T_{N E T}$ is the Network life, $N_{R}$ is the numbers of rounds for which CHs communicated with sink and $t_{r}$ is the individual round time 


\subsubsection{Cluster Time: Intra Cluster Time}

Time allotted for each cluster to sense and transmit to its $\mathrm{CH}$, $t_{c l}$ is determined as,

$$
t_{c l}=\left(t_{1} \times n_{a}\right)
$$

where, $t_{c l}$ is the total time allotment for one cluster, intra cluster time, $t_{1}$ is the time for one active node to sense and transmit to $\mathrm{CH}$ and $n_{a}$ is the number of active nodes in cluster (cluster time mainly depends on $n_{a}$, more number of active nodes, more time allotted for the cluster)

$$
T_{c l}=\sum_{1}^{n_{c}} t_{c l}
$$

where, $T_{c l}$ is the total intra cluster time and $n_{c}$ is the number of clusters formed in one round

\subsubsection{Cluster Time: Inter Cluster Communication Time}

Total time required for all $\mathrm{CHs}$ to transmit data to sink $t_{c o m}$, is determined as,

$$
t_{\text {com }}=t_{2} \times n_{c-}
$$

where, $t_{\text {com }}$ is the inter cluster communication time, $t_{2}=$ time for $\mathrm{CH}$ to forward cluster data to sink and $n_{c}=$ number of clusters formed in one round

\subsubsection{Round Time:}

Time required for all active nodes to sense and transmit the sensed data to sink through $\mathrm{CH}, t_{r}$

$$
t_{r}=\left(T_{c l}+t_{c o m}\right)
$$

where, $t_{r}=$ round time

\subsubsection{Distance between Two Nodes:}

Distance between any two nodes is calculated using Euclidian Eq.(6) as follows,

$$
d(x, y),(a, b)=\sqrt{ }(x-a)^{2}+(y-b)^{2}
$$

where, $d$ is the distance between two nodes, $(x, y)$ is the coordinates of node- $n_{1}$ and $(a, b)$ is the coordinates of node- $n_{2}$

\subsubsection{Energy Calculations: Intra Cluster Communication Energy}

Energy spent for sensor node sensing and communication to $\mathrm{CH}$ in every round, $E_{\text {INTRA-cluster/round }}$ is calculated as,

Energy usage/node $=$ energy dissipation during (receiving + transmitting + sensing + overhearing + sleeping + idling).

$$
E_{T S \text {-node }}=E_{s}+E_{i}+E_{s l}+E_{o h}+E_{t x}+E_{r x}+E_{m}
$$

where, $E_{T S \text {-node }}$ is the Total energy spent in one round for intra cluster communication /node, $E_{s}$ is the Energy spent on sensing, $E_{i}$ is the Energy spent in idle period, $E_{s l}$ is the Energy spent in sleep period, $E_{o h}$ is the Energy spent on overhearing, $E_{t x}$ is the Energy spent for transmitting data/byte/meter, $E_{r x}$ is the Energy spent in receiving data/byte/meter and $E_{m}$ is the Energy spent for mobility when compared to $E_{t x}, E_{r x}, E_{m}$, the others can be neglected,

$$
E_{T S-n o d e}=E_{t x}+E_{r x}+E_{m}
$$

where, $E_{T S \text {-node }}$ is the total transmission energy/node

$$
E_{T S \text {-cluster } / \text { round }}=\left(E_{T S \text {-node }} \times n_{a}\right)
$$

where, $E_{T S-c l u s t e r}$ is the total transmission energy used per cluster/ round and $n_{a}$ is the number of active nodes in a cluster
Energy spent to communicate to $\mathrm{CH}$ is calculated using Eq.(10). As every intermediate node has to receive and transmit we have

$$
E_{T r-n o d e}=E_{t x}+E_{r x}
$$

where, $E_{T r \text {-node }}$ is the total energy used for receiving and transmitting/node

$$
E_{T r \text {-cluster/round }}=\left(\left(E_{T r \text {-node }} \times n_{r}\right)+E_{r x(C H)}\right)
$$

where, $E_{T r \text {-cluster/round }}$ is the total energy used for receiving/cluster/ round, $n_{r}$ is the number of nodes participated in reception/cluster ( $n_{a \neq} n_{r}$, as multi hop used for intra communication)

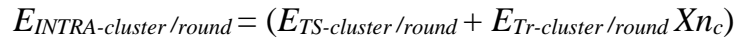

\subsubsection{Inter Cluster Communication Energy:}

Energy spent for communication from $\mathrm{CH}$ to sink in every round, $E_{C H-S I N K}$ is calculated as,

$$
E_{T S R-I N T E R}=\left(E_{S}\left(T_{X}+R_{X}\right)\right) N_{P}+E_{t x} \_C H
$$

where, $E_{T S R-I N T E R / \text { round }}$ is the total energy spent in one round for inter cluster communication, $E_{S}\left(T_{X}+R_{X}\right)$ is the Energy spent for transmission and reception by participating intermediate nodes, $N_{P}$ is the total number of nodes taking part in $\mathrm{CH}$ to sink communication, $E_{t x \_}{ }_{C H}$ is the energy spent by $\mathrm{CH}$ to communicate to next intermediate node

\subsubsection{Total Energy spent in One Round:}

Total energy spent to complete one round is summation of energy spent for intra and inter cluster communication, calculated as $E_{\text {total }}$ and Total energy spent in one round = Eq.(11) + Eq.(12)

$$
E_{\text {total }}=E_{T S-\text { cluster } / \text { round }}+E_{T r \text {-cluster } / \text { round }}+E_{T S R-I N T E R / \text { round }}
$$

where, $E_{\text {total }}$ is the Total energy spent in one round

By Eq.(13), total energy spent in every round is summation of intra and inter cluster energy spent. Number of $T x$ nodes in every cluster is kept minimum by using selective sensing, this helps in reducing energy spent in intra cluster communication. As intra cluster communication time allotment is also done considering the amount of $T x$ nodes only. Results in protocol being energy efficient.

\section{RESULTS AND DISCUSSION}

NS2 simulation tools are used to test and evaluate the proposed algorithm. The setup for simulation of the Energy Efficient Centralized Dynamic Clustering Selective Sensing Collision Free Dynamic Reservation MAC Protocol for MWSN are summarized in Table.1. Mobile nodes use random mobility with a pause between mobility and sink moves inside the field on fixed path.

Table.1. Simulation setup

\begin{tabular}{|l|c|}
\hline \multicolumn{1}{|c|}{ Parameters } & Values \\
\hline Number of nodes & 45,80 and 200 \\
\hline Simulation area & $250 \times 250$ and \\
& $500 \times 500 \mathrm{mts}$ \\
\hline Sensor node deployment & Random \\
& deployment \\
\hline Number of sink & 1 \\
\hline
\end{tabular}




\begin{tabular}{|l|c|}
\hline Sink path & fixed \\
\hline Mobility for nodes & $\begin{array}{l}\text { Random } \\
\text { mobility }\end{array}$ \\
\hline $\begin{array}{l}\text { Power consumption in node } \\
\text { for mobility/meter }\end{array}$ & $0.01 \mathrm{~mW}$ \\
\hline $\begin{array}{l}\text { power consumption for } \\
\text { sending the data/byte/meter }\end{array}$ & $0.000005 \mathrm{~mW}$ \\
\hline $\begin{array}{l}\text { power consumption for } \\
\text { receiving the data/byte/meter }\end{array}$ & $0.000001 \mathrm{~mW}$ \\
\hline Transmission technology & wireless RF \\
\hline Initial energy of sensor nodes & $100 \mathrm{~J}$ \\
\hline
\end{tabular}

Table.2. Sensor Field Setup

\begin{tabular}{|c|c|l|l|}
\hline $\begin{array}{c}\text { Sensor } \\
\text { field } \\
\text { area }\end{array}$ & $\begin{array}{c}\text { Sensor } \\
\text { deployment }\end{array}$ & Sink deployment & Sink type \\
\hline $250 \times 250$ & $\begin{array}{l}\text { a) } 45 \\
\text { b) } 80\end{array}$ & $\begin{array}{l}\text { a) Outside and inside } \\
\text { b) inside }\end{array}$ & $\begin{array}{c}\text { Stationary } \\
\text { Mobile }\end{array}$ \\
\hline $500 \times 500$ & 200 & outside & Stationary \\
\hline
\end{tabular}

The algorithm is tested for different setups of Table.2. Sensor field in divided into square shaped virtual grids. The number of grids calculated as in reference [16], Determines optimal number cells to be $5 \%$ of total number of sensor nodes, calculated by,

$$
\text { Number of cells, } k \text { is } 0.05 \mathrm{~N}
$$

where, $N$ is the number of sensor nodes and $N=45,80$ and 200 are used (Table.2) to evaluate the performance of algorithm with mobile sensor nodes and following sink combination, 1) Mobile sensor nodes-stationary sink and 2) Mobile sensor nodes-Mobile sink.

The performance of algorithm is compared with VGDRA, shown in Fig.6, where MS moves outside sensor field. In most approaches the latest location of mobile sink (MS) is flooded to entire sensor field this incurs a large amount of energy consumption, therefore avoided in this work. As sink follows a fixed path and $\mathrm{CHs}$ communicate to it in reserved slots, this work requires no $\mathrm{MS}$ location updates. $\mathrm{CHs}$ follow the following propagation rules,

1. Mobile sink travel to and fro on fixed path at the center of sensor field and

2. CHs of cells on LHS of route transmit data when MS is travelling in forward direction.

a. CHs adjacent to path transmit first

b. $\mathrm{CHs}$ in far cell transmit after all $\mathrm{CHs}$ of adjacent cell completes their data transfer.

c. Steps ' $a$ ' and 'b' are repeated till all CHs on LHS cells complete their data transfer.

3. MS travels in reverse direction

4. Step 2 is repeated for CHs in cell on RHS of the path.

Hence routing adjustment for mobile sink in this work incurs least communication cost viva optimal routes to the mobile sink. These paths are built using $\mathrm{CHs}$ and nodes in the path from $\mathrm{CH}$ to mobile sink.

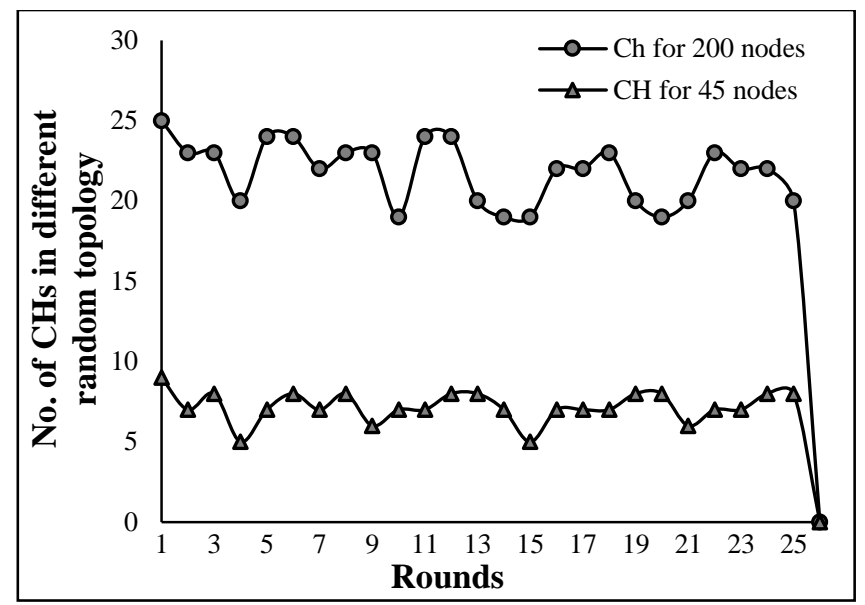

Fig.4. Number of CHs formed

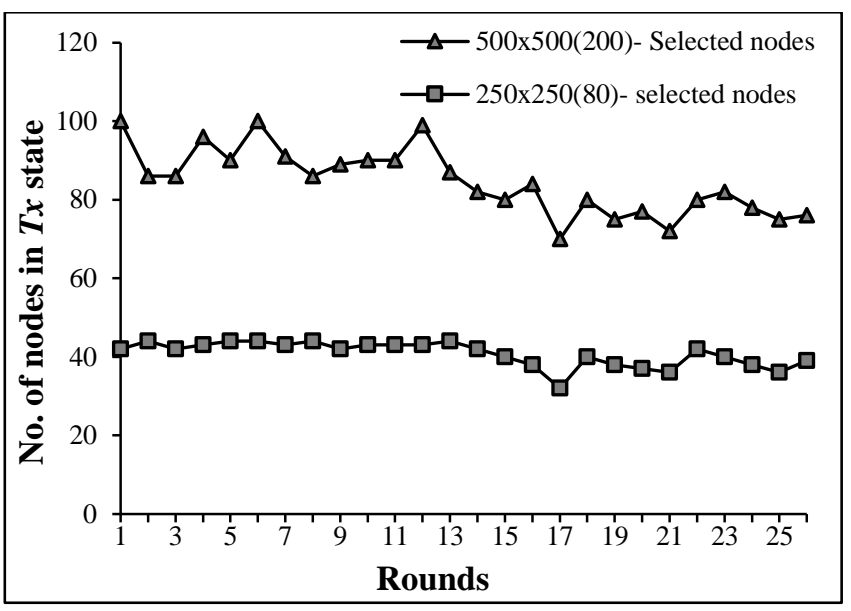

Fig.5. Number of selected nodes vs. Rounds

In Fig.7, 80 nodes and one MS are deployed in an area of $250 \times 250$ and partitions the sensor field into $4 \times 4$ virtual grid. MS path is fixed on the center grid as shown in figure. The $\mathrm{CHs}$ on LHS is 1, 2, 5, 6, 9, 10, 13, 14 shown in Fig.7, of the path transmit data when sink travels in forward direction. First CHs in cell-1 transmit and then $\mathrm{CHs}$ of cell-2 transmit data. This repeats for rest LHS cells. Whereas CHs on RHS of the path 3, 4, 7, 8, 11, 12, 15, 16 on Fig.7, transmit to sink while it travels in reverse direction. First CHs in cell 15 transmits data to sink and then CHs of cell 16, this repeats for all RHS cells on RHS of path Fig.7.

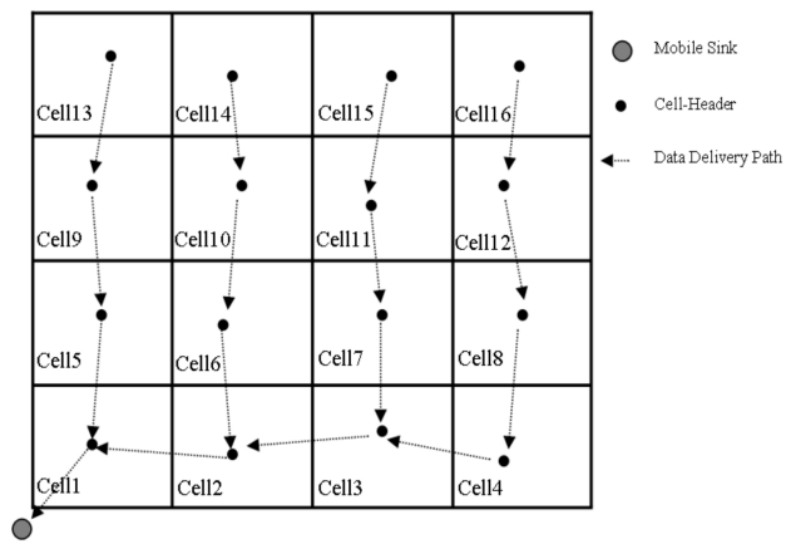




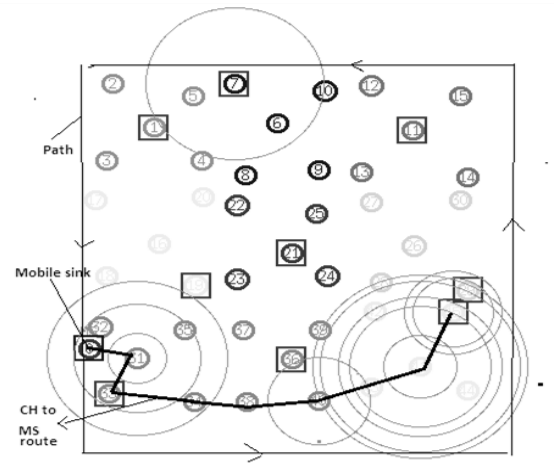

Fig.6. Paths CH to MS VGDRA
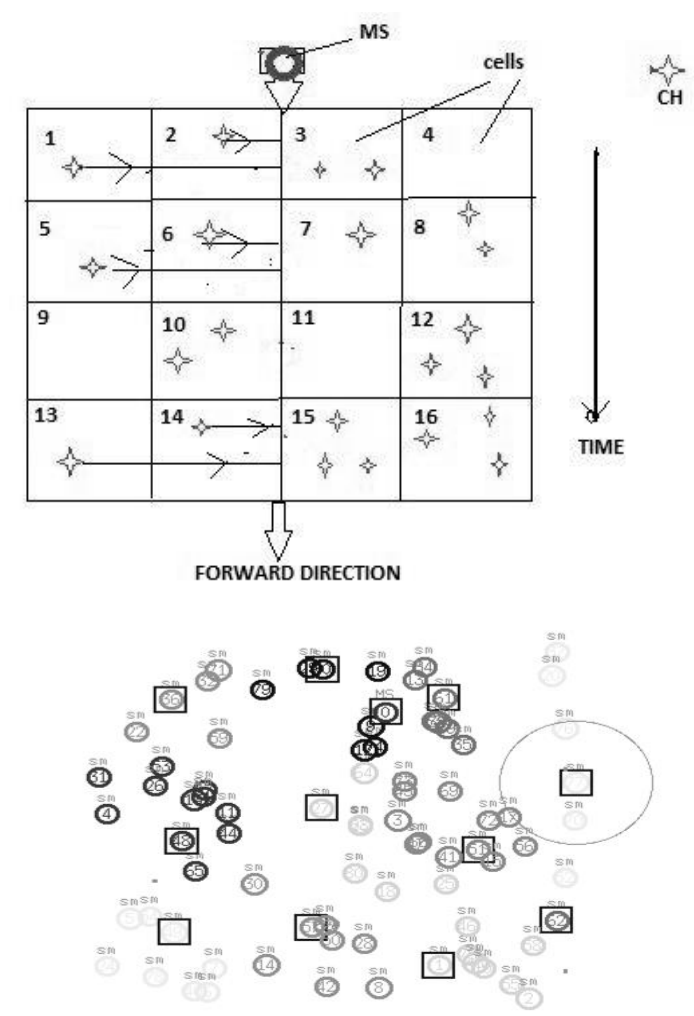

Fig.7. Paths $\mathrm{CH}$ to MS

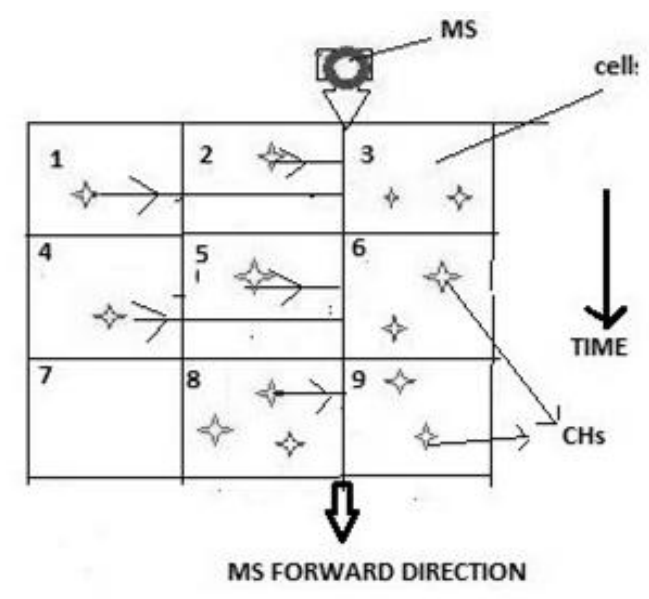

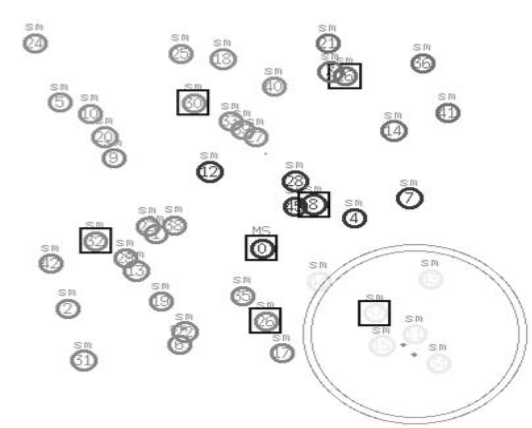

Fig.8. Paths $\mathrm{CH}$ to MS

In Fig. 8,45 nodes are deployed in area of $250 \times 250$ and MS path is set grid as shown in figure. Sink collects data from LHS cells similar to Fig.7 in forward journey but in return journey collects from cell-9, 6 and 3 only.

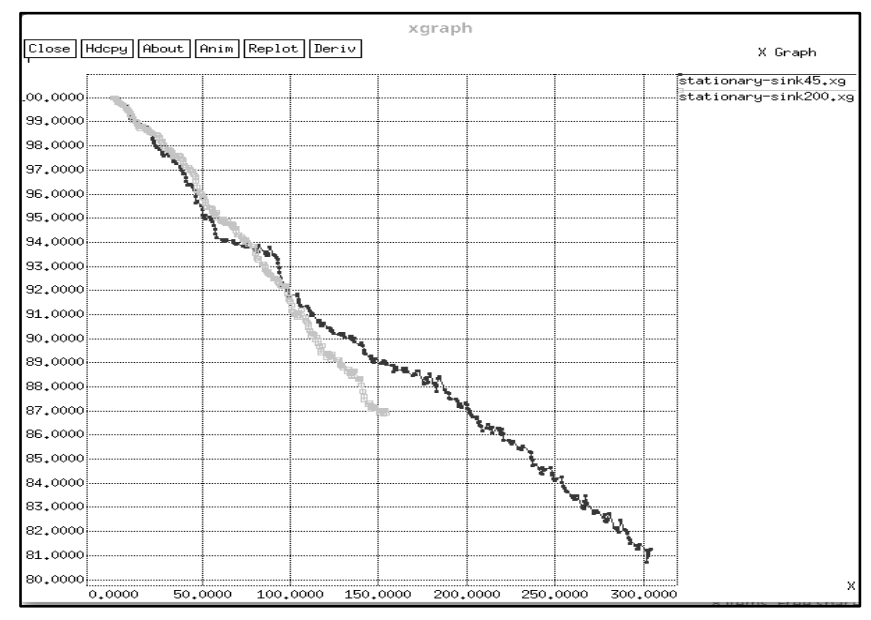

Fig.9. Stationary sink Residual energy

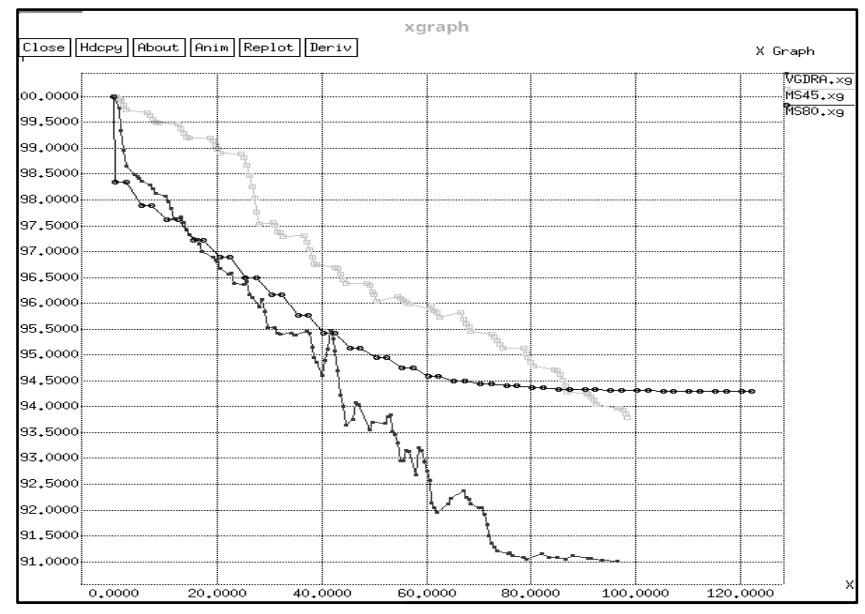

Fig.10. Mobile sink Residual energy 


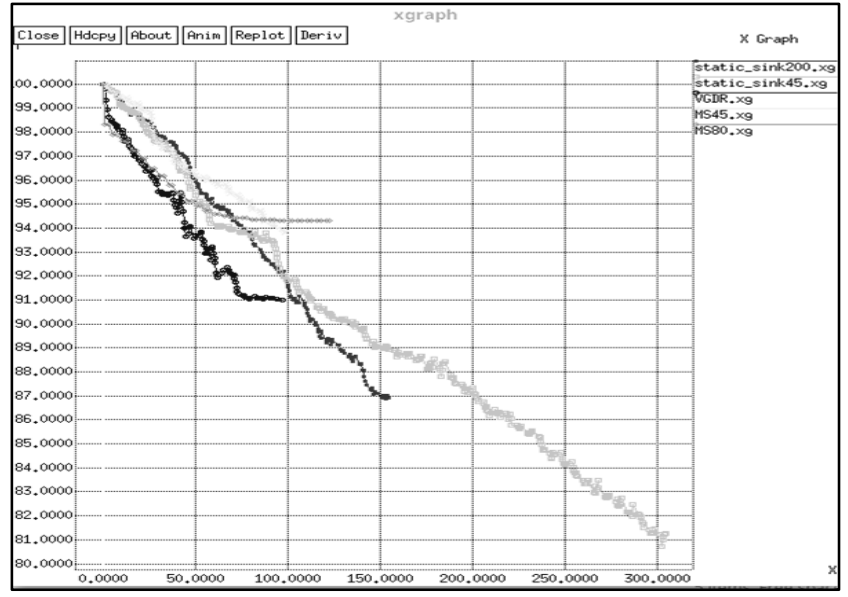

Fig.11. Comparing residual energy for stationary and mobile sink

Nodes energy is spent for performing different activities, is the sum of energy spent for sensing, data processing, communication and mobility. This work concentrates on the energy saving in sensing process and $\mathrm{CH}$ to sink communication.

The Fig.9 compares residual energy of proposed algorithm for 45 and 200 nodes using stationary sink placed outside sensor field. It is observed that initially the performance of both deployments is almost same. But after time $>100$, residual energy is less due to more energy expenditure as deployment area is more. The Fig.10 compares residual energy of VGDRA and proposed algorithm for 45 and 80 nodes using MS.

In VGDRA sensor nodes are stationary and only sink is in motion on fixed path outside. Here all nodes are allowed to transmit data to $\mathrm{CH}$ hence intra cluster communication is more and collusions of data packet is also more as $\mathrm{CHs}$ are allowed to transmit simultaneously. The proposed algorithm with mobile sensors and MS performance is better than VGDRA as many nodes are in No_Tx state, and inter cluster communication cost is reduced to half the cost as sink travels inside the field.

The Fig.11 compares residual energy of proposed algorithm for stationary sink placed outside and mobile sink traveling inside. As discussed proposed algorithms performance is found better than VGDRA and with stationary sink.

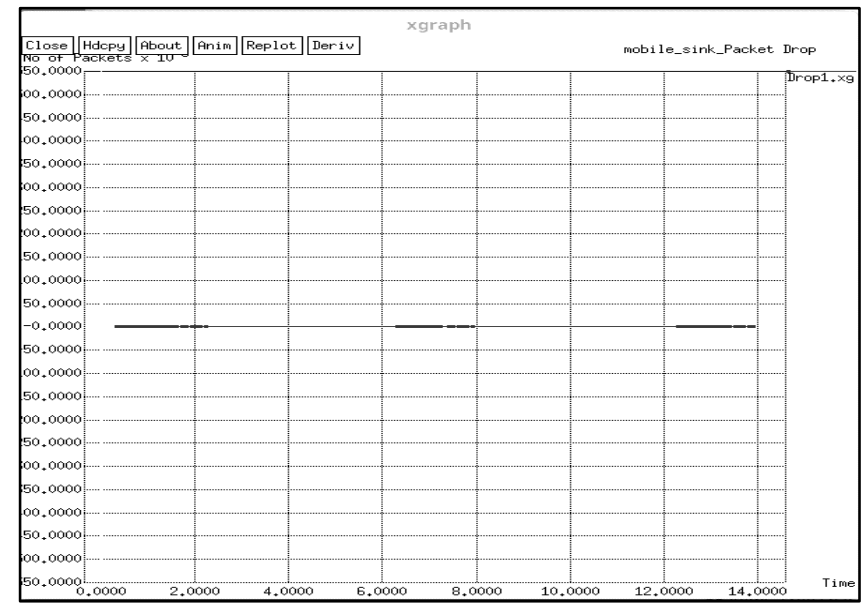

(a) Proposed

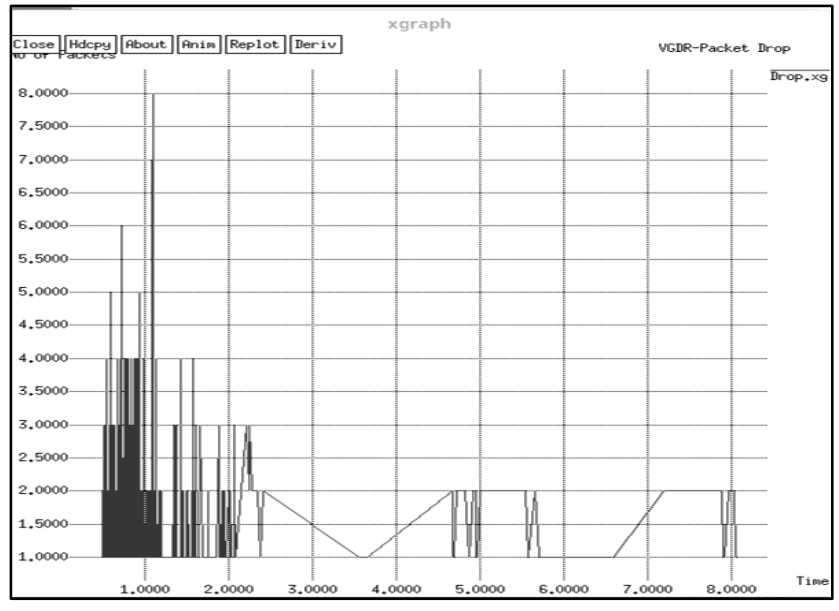

(b)VGDRA

Fig.11. Comparing packet drops

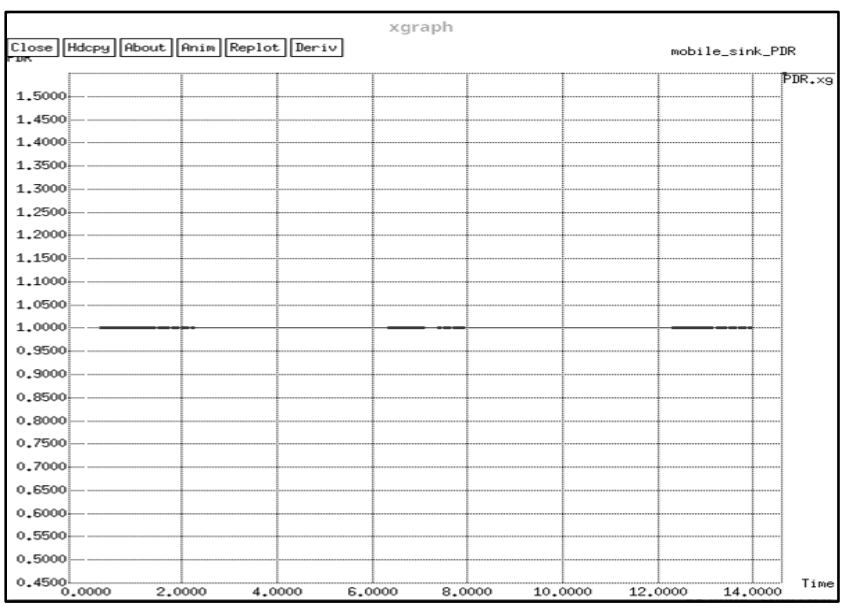

(a) Proposed

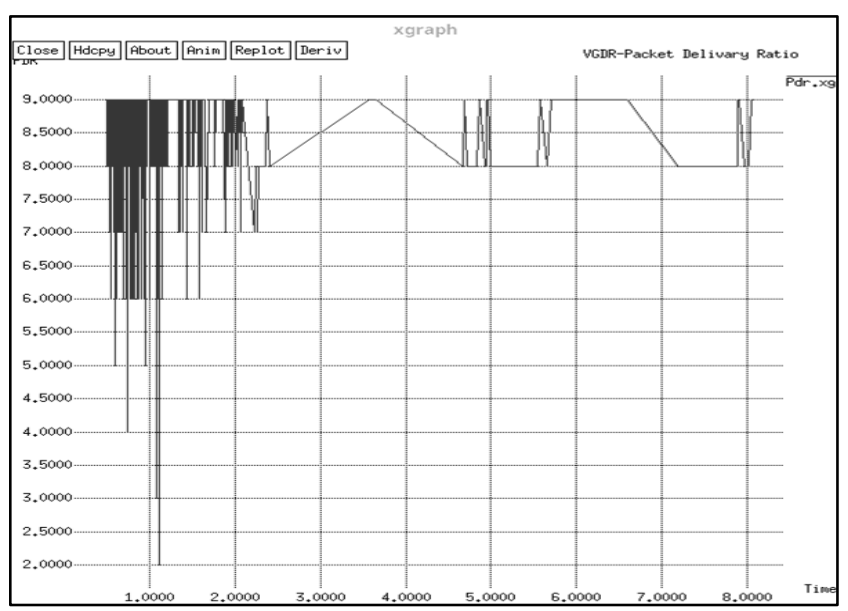

(b) VGDRA

Fig.12. Comparing PDR

The Fig.11 shows the data packet drop of proposed work is zero as communication from source to sink is carried out in reserved time slots. The Fig.11(a) shows the payload transmission 
due to this proposed protocols ' is collusion free, resulting in zero packet drop. In case of VGDRA in Fig.11(b), packet drop range is $10 \%$ to $20 \%$. From, Fig.12(a) and Fig.12(b), it is observed the proposed algorithm packet delivery ratio is $100 \%$ and for VGDRA it is $80 \%$ to $90 \%$. This clearly demonstrates that proposed algorithm is collision free.

\section{CONCLUSION}

This paper proposes an energy efficient collusion free clustering algorithm for mobile sensor networks which comprises dynamic clustering and dynamic reservation for delay tolerant and high integrity MWSNs. The performance of this protocol can be further studied using MS with random path. Dynamic clustering consists of two components, one mechanism selects sensors from grids using nodes proximity and other decides sensing schedule for every node in cluster and forms varying size clusters. Dynamic reservation mechanism schedules both intra and inter communication time considering number of clusters and cluster size in every round. As entire operational load is taken up by sink, sensors only perform minimum and essential work spending less energy. It is observed that when sensing area is large, more energy is consumed for inter cluster communication. The residual energy of network is more when compared to stationary sink placed outside sensing field, as mobile sink saves energy used for inter cluster communication. As reservation MAC is used for communication PDR is observed to be $100 \%$ as packet drops are absent. By using both selective sensing and mobile sink the algorithms' performance is found better than VGDRA and while using stationary sink. Performance evaluation is carried extensively through simulation. Results show the proposed dynamic centralized clustering algorithm is more energy efficient.

\section{REFERENCES}

[1] Chaojie $\mathrm{Xu}$, Zhongling Liu, Jun Xia and Hui Yu, "An Adaptive Distributed Re-Clustering Scheme for Mobile Wireless Sensor Networks", Proceedings of International Conference on Wireless Communications and Signal Processing, pp. 1-6, 2015.

[2] W.R. Heinzelman, A. Chandrakasan and H. Balakrishnan, "Energy Efficient Communication Protocol for Wireless Micro Sensor Networks", Proceedings of IEEE 33 ${ }^{\text {rd }}$ Annual Hawaii International Conference on System Sciences, 2000.

[3] O. Younis and S. Fahmy, "Heed: A Hybrid, EnergyEfficient, Distributed Clustering Approach for Ad Hoc Sensor Networks", IEEE Transactions on Mobile Computing, Vol. 3, No. 4, pp. 366-379, 2004.

[4] Getsy S. Sara, R. Kalaiarasi, S. Neelavathy Pari and D. Sridharan, "Energy Efficient Clustering and Routing in Mobile Wireless Sensor Network", International Journal of Wireless and Mobile Networks, Vol. 2, No. 4, pp. 1-9, 2010.

[5] R. Anitha and P. Kamalakkannan, "Eedbc-m: Enhancement of Leach Mobile Protocol with Energy Efficient Density- based Clustering for Mobile Sensor Networks", International Journal of Computer Applications, Vol. 74, No. 14, pp. 19-27, 2013.

[6] Changlin Ma, Nian Liu, Yuan Ruan, “An Energy-efficient Clustering Algorithm in Mobile Sensor Networks", Proceedings of IEEE International Conference on Signal Processing, Communication and Computing, pp. 1-4, 2013.

[7] Changlin Ma, Yuan Ruan and Huajing Fang, "Optimal Design of Mobile Sensor Networks based on Resource Constraint and Mobility", Proceedings of $5^{\text {th }}$ International Conference on Intelligent Human-Machine Systems and Cybernetics, Vol. 2, pp. 360-363, 2013

[8] Hyunsook Kim and Gyeongsan, "Cluster Head Selection Scheme for Minimizing the Changes of the Cluster Members considering Mobility in Mobile Wireless Sensor Networks", Proceedings of $15^{\text {th }}$ International Conference on Advanced Communication Technology, pp. 285-289, 2013.

[9] M. Zuniga and B. Krishnamachari, "Analyzing the Transitional Region in Low Power Wireless Links", Proceedings of First Annual IEEE Communications Society Conference on Sensor and Ad Hoc Communications and Networks, pp. 517-526, 2004.

[10] Qingquan Zhang, Lingkun Fu, Yu Jason Gu, Lin Gu, Qing Cao, Jiming Chen and Tian He, "Collaborative Scheduling in Dynamic Environments using Error Inference", IEEE Transactions on Parallel and Distributed Systems, Vol. 25, No. 3, pp. 591-601, 2014.

[11] Yu Gu, Jianwei Niu, Tian He and David Hung-Chang Du, "Achieving Asymmetric Sensing Coverage for Duty Cycled Wireless Sensor Networks", IEEE Transactions on Parallel and Distributed Systems, Vol. 25, No. 12, pp. 3076-3087, 2014.

[12] Vinicius Galvao Guimaraes, Adolfo Bauchspiess, Renato Mariz de Moraes, "Dynamic Timed Energy Efficient and Data Collision Free MAC Protocol for Wireless Sensor Networks", IEEE Latin America Transactions, Vol. 13, No. 2, pp. 416-421, 2015.

[13] Ruitao Xie and Xiaohua Jia, "Transmission-Efficient Clustering Method for Wireless Sensor Networks using Compressive Sensing", IEEE Transactions on Parallel and Distributed Systems, Vol. 25, No. 3, pp. 806-815, 2014.

[14] Christian Bettstetter, Hannes Hartenstein and Xavier PerezCosta, "Stochastic Properties of the Random Waypoint Mobility Model", Wireless Networks, Vol. 10, No. 5, pp. 555-567, 2004.

[15] Ossama Younis, Marwan Krunz and Srinivasan Ramasubramanian, "Node Clustering in Wireless Sensor Networks: Recent Developments and Deployment Challenges", IEEE Network, Vol. 20, No. 3, pp. 20-25, 2006.

[16] Abdul Waheed Khan, Abdul Hanan Abdullah, Mohammad Abdur Razzaque and Javed Iqbal Bangash, "VGDRA: A Virtual Grid-Based Dynamic Routes Adjustment Scheme for Mobile Sink-Based Wireless Sensor Networks", IEEE Sensors Journal, Vol. 15, No. 1, pp. 526-534, 2015. 